\title{
Identification of a Guinea Pig Fc $\gamma$ Receptor that Exhibits Enhanced Binding to Afucosylated Human and Mouse IgG
}

\author{
Changchuin Mao, Richard Near and Wenda Gao*
}

Antagen Pharmaceuticals, Inc. Boston, MA 02118, USA

\begin{abstract}
Glyco-engineered recombinant antibodies are currently being developed as the next generation therapeutics to treat human diseases, including cancer, autoimmunity and infection. Antibodies lacking core fucosylation show great increase in affinity for FcyRIIIA, leading to an improved receptor-mediated effector function. While afucosyl human IgG1 exhibits 50-100-fold increase in antibody-mediated cellular cytotoxicity (ADCC), a key immune effector mechanism underlying the anti-cancer effect of some approved therapeutic antibodies, it is not clear whether such glyco-engineered antibodies would find similar use for infectious disease. Due to the species difference, human antibodies may have different binding properties towards corresponding Ig $\mathrm{G}$ receptors from animals used for modeling infection and intoxication. During the course of studying a recombinant human IgG1 in neutralizing diphtheria toxin (DT) in Guinea pigs (Cavia porcellus), we identified a previously uncharacterized Guinea pig protein HOVDZ8 from UNIPROT database that shows high sequence homologies to human FcyRIIIA and mouse FcyRIV. This Fcy receptor, which we named as gpFcyRIV, also demonstrates functional similarity although not to the same extent as the human and mouse counterparts, in that it binds to afucosyl human and mouse $\lg \mathrm{G}$ much stronger than to the wild type antibodies. Thus, Guinea pigs can be used to compare the efficacies of wild type vs. afucosyl anti-DT human IgG1 in toxin removal and animal protection. Molecular and functional characterization of human FcyRIIIA and mouse FcyRIV equivalents in other species could expand the list of preclinical animal models for testing afucosyl human antibodies in treating various human diseases.
\end{abstract}

Keywords: Immunoglobulin G; Fc receptor; Afucosylation; Guinea pig

\section{Abbreviations}

CHO: Chinese Hamster Ovary; ADCC: Antibody-dependent Cellular Cytotoxicity; ADCP: Antibody-dependent Cellular Phagocytosis, Fut8: Alpha-(1,6)-fucosyltransferase 8; TALEN: Transcription Activator-like Effector Nuclease

\section{Introduction}

Monoclonal antibodies (Mabs) are being developed as therapeutics for treating various human diseases. The vast majority of marketed IgG Mabs are produced in mammalian cells, especially Chinese hamster ovary $(\mathrm{CHO})$ cells. IgG1 antibodies produced from wild type $\mathrm{CHO}$ cells have a fucose residue in a 1,6 linkage to the first GlcNAc of the oligosaccharide core ("core fucosylation") in their bi-antenna glycan attached to asparagine (Asn) residue 297 in the Fc region. It has been well established by many laboratories that the addition of core fucose diminishes the affinity of IgG Fc to the human FcyRIIIA receptor expressed on natural killer cells, macrophages, neutrophils, and other immune cells. As a result, IgG molecules lacking a core fucose residue bind with 50-fold enhanced affinity to Fc $\gamma$ RIIIA and exhibit 50-100-fold greater cellular immune functions, for example, antibodydependent cellular cytotoxicity (ADCC) and antibody-dependent cellular phagocytosis (ADCP) [1].

Currently, afucosyl antibodies with enhanced ADCC towards target cells are mainly being pursued in the immuno-oncology area. For example, Mogamulizumab is an afucosyl humanized Mab targeting CC chemokine receptor 4 (CCR4), approved in Japan in 2012 for the treatment of relapsed or refractory adult T-cell leukaemia-lymphoma (ATL) [2]. Obinutuzumab against CD20 is the second afucosyl Mab approved by US FDA in 2013 for treating B cell lymphoma [3]. Besides these two approved, more afucosyl antibodies are in the research pipeline, and the pharmaceutical industry has recognized the advantages of fucose-free therapeutic antibodies [3]. While the benefits of afucosyl Mabs in cancer immunotherapy are obvious, so far there are only two reports in infectious disease area supporting their potential application, where afucosyl antiviral antibodies show enhanced cellmediated antiviral potency against the HIV and Ebola viruses [4,5].

The main reason for this deficiency is due to the lack of knowledge on the binding properties of human IgGs with or without fucose towards corresponding antibody receptors from animals used for modeling infection and intoxication. Among the human Fcy receptors, only Fc $\gamma$ RIII is sensitive to IgG defucosylation. In mice, mFc $\gamma$ RIV is regarded as the functional homologue of hFc $\gamma$ RIIIA. Accordingly, only $\mathrm{mF} c \gamma \mathrm{RIV}$ is sensitive to IgG defucosylation among all the murine $\mathrm{Fc} \gamma$ receptors [6]. Little is known on the counterparts of hFc $\gamma$ RIIIA and $\mathrm{mF} c \gamma \mathrm{RIV}$ in other species, as well as their binding sensitivity towards afucosyl antibodies.

During the course of studying a human anti-diphtheria toxin IgG1 Mab in Guinea pigs (Cavia porcellus), we first asked: 1) whether hIgG1 can bind to Guinea pig Fc $\gamma R$, and 2) whether there is an equivalent of $\mathrm{hFc} \gamma \mathrm{RIIIA}$ and $\mathrm{mFc} \gamma \mathrm{RIV}$ in Guinea pigs that exhibits enhanced binding to afucosyl hIgG1. Our studies show that a previously uncharacterized Guinea pig protein H0VDZ8 from UNIPROT database is a structural and functional homologue to human Fc $\gamma$ RIIIA and mouse Fc $\gamma$ RIV.

*Corresponding author: Wenda Gao, Antagen Pharmaceuticals, Inc., 650 Albany St., STE 112, Boston, MA 02118, USA, Tel: 6176380315; E-mail: wendagao01@gmail.com

Received: September 13, 2016; Accepted: September 19, 2016; Published: September 26, 2016

Citation: Mao C, Near R, Gao W (2016) Identification of a Guinea Pig Fcy Receptor that Exhibits Enhanced Binding to Afucosylated Human and Mouse IgG. J Infect Dis Med 1: 102. doi: 10.4172/2576-1420.1000102

Copyright: (c) 2016 Mao C, et al. This is an open-access article distributed under the terms of the Creative Commons Attribution License, which permits unrestricted use, distribution, and reproduction in any medium, provided the original author and source are credited. 
Citation: Mao C, Near R, Gao W (2016) Identification of a Guinea Pig Fcy Receptor that Exhibits Enhanced Binding to Afucosylated Human and Mouse IgG. J Infect Dis Med 1: 102. doi: 10.4172/2576-1420.1000102

\section{Materials and Methods}

Cell line generation: $\mathrm{CHO}$ cell lines expressing the functional hFc $\gamma$ RIIIA, mFc $\gamma$ RIV or gpFcRIV complexes on the cell surface were generated with the "Toggle-In" CHO system (Antagen Pharmaceuticals, Inc., Boston, MA). The establishment of the anchor "Toggle-In" CHO cell line and the construction of pTOG3 and pTOG4 expression vectors with alternate use of Hygromycin B and Puromycin as selection markers will be published in more detail elsewhere. Briefly, an anchor "ToggleIn" CHO cell line was selected by FACS sorting on d1EGFP expression at a $\mathrm{CHO}$ genomic "hot-spot". Several genes involved in enhancing protein expression, e.g., SRP54, SRP9, SRP14, ERO1-L and FGF9 were sequentially integrated at this "hot-spot" by replacing d1EGFP with Cre-LoxP recombination-mediated cassette exchange (RMCE). A $\mathrm{CHO}$ cell line thus generated is named as the master "Toggle-In" line, which has been used for establishing other cell line models. Herein for example, human FcR common $\gamma$ chain in pTOG4 was first transfected into the master "Toggle-In" line, and selected with Puromycin (10 $\mu \mathrm{g} / \mathrm{mL}$ ). An FcR $\gamma$-positive clone was picked and further transfected with hFc $\gamma$ RIIIA-V158, mFc $\gamma$ RIV or gpFc $\gamma$ RIV, all cloned in pTOG3, respectively, and selected with Hygromycin B $(1 \mathrm{mg} / \mathrm{mL})$. Single CHO clones of double transfectants were screened and confirmed by FACS staining with hIgG1 or mIgG2a.

Expression of wild type and afucosyl antibodies: A ScFv-hIgG1 construct, where its $\mathrm{ScFv}$ part utilizes anti-TNF $\alpha$ Humira sequence to fuse with human IgG1 Fc, was cloned into pDirect4.0 expression vector (Antagen), and transfected into either wild type or proprietary Fut8/- CHO-K1 cells (Antagen). This Fut8-/- CHO cell line was established in-house with TALEN technology. Detailed method and genomic sequences of the two TALEN-targeted Fut8 alleles will be disclosed elsewhere. A mouse hybridoma secreting IgG2a antibody (PK136, ATCC, Manassas, Virginia) against mouse NK1.1 was transfected with two CRISPR constructs targeting the mouse Fut8 gene (Antagen). The Fut8-/- CHO or hybridoma cells were selected by MACS depletion of fucose-positive cells, and confirmed by negative staining with FITClabeled Lens culinaris agglutinin (LCA, Vector Laboratories, Inc.,
Burlingame, CA), recognizing the $\alpha-1,6$ fucosylated tri-mannnosyl core structure on $\mathrm{N}$-glycans on the cell surface.

Comparing wild type and afucosyl antibodies in binding to Fc $\gamma \mathrm{R}$ : Both CHO-derived wild type and afucosyl hIgG1, as well as hybridomaderived wild type and afucosyl mIgG2a, were purified with Protein A column from culture supernatants. Protein concentrations were determined by OD280, and titrated amounts of wild type and afucosyl antibodies were assayed for receptor binding with goat anti-human or mouse secondary antibody in flow cytometry, using $\mathrm{CHO}$ cell lines expressing hFc $\gamma$ RIIIA, mFc $\gamma$ RIV or gpFc $\gamma$ RIV.

\section{Results and Discussion}

To search for Guinea pig homologue(s) of hFc $\gamma$ RIIIA and $m F c \gamma R I V$, we blasted UNIPROT database and found a few candidate Guinea pig proteins that show various homologies. One uncharacterized protein H0VDZ8 that has not been assigned a gene name shows the highest homologies: $55.3 \%$ identical and $72.5 \%$ similar amino acids with hFcrRIIIA; $54.9 \%$ identical and $71.4 \%$ similar amino acids with mFc $\gamma$ RIV (Figure 1). H0VDZ8 could be the potential Guinea pig equivalent of hFcrRIIIA and $\mathrm{mFc} \gamma \mathrm{RIV}$.

Based on its amino acid sequence, we ordered full gene synthesis for H0VDZ8 as "gpFc $\gamma$ RIV" with CHO codon optimization (Integrated DNA Technologies, Inc., Coralville, Iowa), and cloned it into the pTOG3 vector (Antagen). As FcR common $\gamma$ chain co-expression is required for proper protein folding and cell surface display of hFc $\gamma$ RIIIA or $m F c \gamma R I V$, we took the advantage of our "Toggle-In" system (Antagen), where Cre-LoxP based sequential integration of exogenous genes into the same genomic locus is exploited for isogeneic co-expression of the $h F c \gamma R I I I A+h F c R \gamma$, $\mathrm{mFc} \gamma \mathrm{RIV}+\mathrm{hFcR} \gamma$ or gpFc $\gamma \mathrm{RIV}+\mathrm{hFcR} \gamma$ complex (See Methods).

When titrated amounts of wild type and afucosyl hIgG1 were added to $\mathrm{hFc} \gamma \mathrm{RIIIA}-\mathrm{V} 158$ expressing $\mathrm{CHO}$ cells, afucosyl hIgG1 demonstrated much better binding than wild type hIgG1. The $\mathrm{EC}_{50}$ of afucosyl hIgG1 binding to $\mathrm{hFc} \gamma \mathrm{RIIIA}-\mathrm{V} 158$ is about $200 \mathrm{ng} / \mathrm{mL}(1.25 \mathrm{nM})$, whereas that of the wild type hIgG1 is $>2000 \mathrm{ng} / \mathrm{mL}(12.5 \mathrm{nM})$ (Figure 2$)$. We estimate that by our method, there is $>10-20$-fold increase in hIgG1 binding to

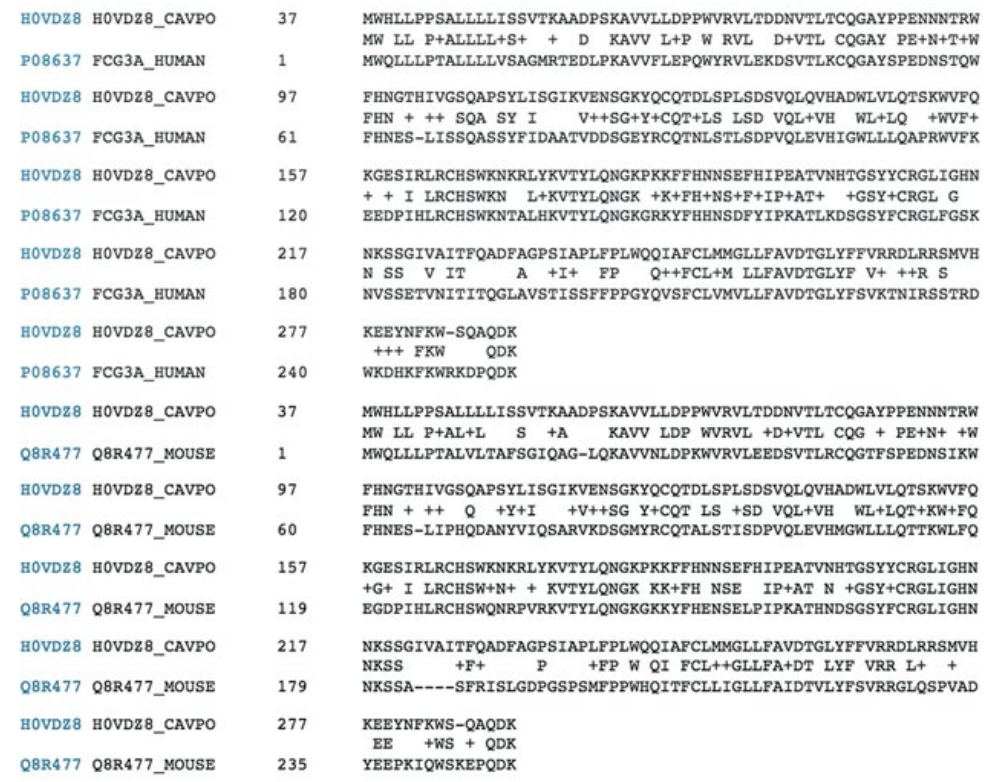

Figure 1: Alignment of Guinea pig protein H0VDZ8 with hFcyRIIIA (P08637, upper) or mFcyRIV (Q8R477, lower). 

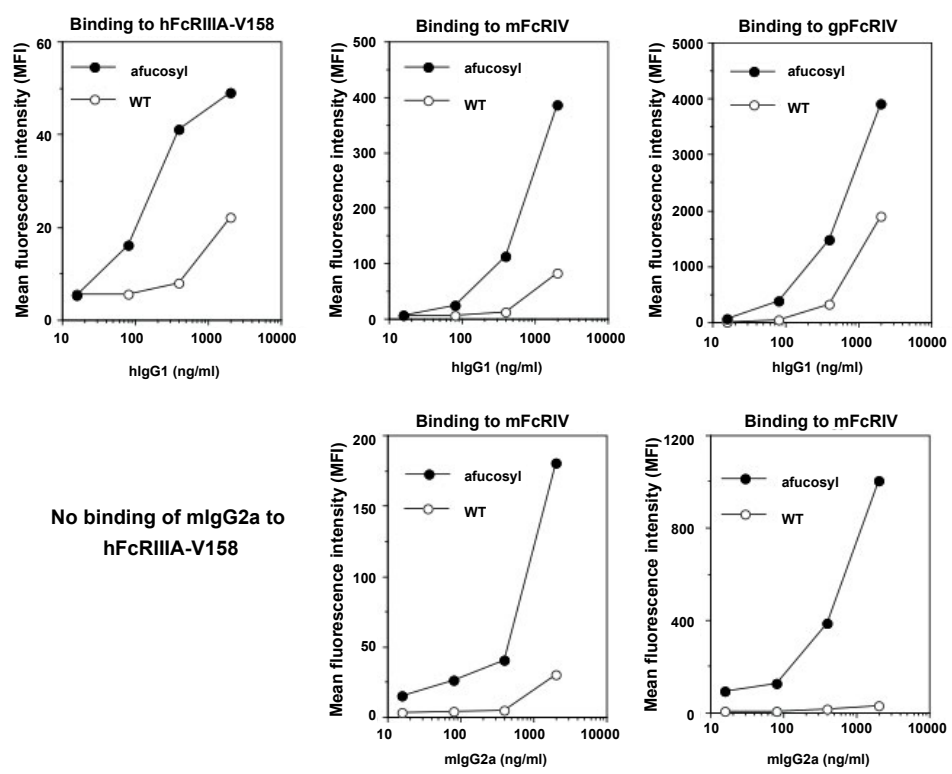

Figure 2: Flow cytometry analysis of binding of wild type or afucosyl hlgG1 (upper) and mlgG2a (lower) to hFcyRIIIA, mFcyRIV or gpFcyRIV (HOVDZ8). Data are representative of two similar experiments.

hFc $\gamma$ RIIIA-V158 after defucosylation. Mouse IgG2a did not show any binding to hFc $\gamma$ RIIIA (Figure 2), emphasizing that species difference will have to be taken into consideration when directly testing in vitro ADCC activities of mouse antibodies using hFc $\gamma$ RIIIA-expressing effector cells.

Interestingly, when wild type and afucosyl hIgG1 or mIgG2a antibodies were added to $\mathrm{mF} c \gamma \mathrm{RIV}$-expressing $\mathrm{CHO}$ cells, very similar binding patterns were obtained (Figure 2). This suggests that murine immune system could largely recapitulate the benefits of hIgG1 defucosylation. In other words, to demonstrate the enhanced therapeutic values of afucosylated humanized murine antibody (hIgG1 isotype) in mouse models of disease, researchers can directly take the advantage of CRISPR knockout of mouse Fut8 gene in hybridoma, and compare hybridoma-derived wild type and afucosyl parental mouse antibodies if they are of ADCC-enabling IgG2a/2b/2c isotypes. This could be an alternative to cloning the VH VL genes from the hybridoma and expressing the chimeric antibody genes with hIgG1 Fc in wild type and Fut8-/- CHO cells.

However, the binding of hIgG1 to gpFc $\gamma$ RIV does not show much enhancement after defucosylation (Figure 2). We repeated the assay and got very similar results: The $\mathrm{EC}_{50}$ of afucosyl hIgG1 binding to gpFc $\gamma \mathrm{RIV}$ is about $289.3 \mathrm{ng} / \mathrm{mL}(1.81 \mathrm{nM})$, whereas that of the wild type hIgG1 is $712.1 \mathrm{ng} / \mathrm{mL}$ (4.45 nM) (data not shown), a mere 2.5 fold enhancement. For mIgG2a, although it does not bind to hFc $\gamma$ RIIIA-V158, its binding to gpFc $\gamma$ RIV is dramatically enhanced after defucosylation (Figure 2).

Our studies answered the initial questions that hIgG1 can bind to Guinea pig $\mathrm{Fc} \gamma \mathrm{R}$ and H0VDZ8 is a structural and functional homologue to human Fc $\gamma$ RIIIA and mouse Fc $\gamma$ RIV. In fact, based on the mean fluorescence intensity (MFI) data in flow cytometry, the binding affinity of hIgG1 to this gpFc $\gamma$ RIV is about 100 -fold and 10-fold over the binding affinity of hIgG1 to hFc $\gamma$ RIIIA and mFcRIV, respectively. We believe these are not due to the cell line differences in receptor surface expression because of integration site and copy number variation seen in the conventional transfection method, as our "Toggle-In" system can eliminate such factors by site-specific isogeneic expression of exogenous genes with equal copy number. Perhaps also due to this stronger base line binding of hIgG1 to gpFcyRIV, the difference between the wild type and the afucosyl forms of hIgG1 in binding to gpFc $\gamma$ RIV is not as dramatic. Therefore, the benefit of afucosyl hIgG1 in Guinea pig model could be under-manifested as in humans. From proof-of-concept point of view, it remains to be tested whether murinized human therapeutic IgG with $\mathrm{mIgG} 2 \mathrm{a} / \mathrm{b} / \mathrm{c}$ Fc tail would better exemplify the benefits of afucosyl antibodies in disease models where Guinea pigs are used as host.

\section{Acknowledgement}

This study is supported by an SBIR contract from CDC (Requisition No: 00HCVGGC-2015-89058). We thank Dr. Yen-Ming Hsu of AB Biosciences, Inc. for the gift of mFcyRIV cDNA.

\section{References}

1. Listinsky JJ, Siegal GP, Listinsky CM (2013) Glycoengineering in cancer therapeutics: a review with fucose-depleted trastuzumab as the model. Anticancer Drugs 24: 219-227.

2. Beck A, Reichert JM (2012) Marketing approval of mogamulizumab: a triumph for glyco-engineering. MAbs 4: 419-425.

3. Small S (2013) Approval of obinutuzumab as a breakthrough therapy for chronic lymphocytic leukemia. Clin Adv Hematol Oncol 11: 809-810.

4. Forthal DN, Gach JS, Landucci G, Jez J, Strasser R, et al. (2010) FCglycosylation influences Fcy receptor binding and cell-mediated anti-HIV activity of monoclonal antibody 2G12. J Immunol 185: 6876-6882.

5. Zeitlin L, Pettitt J, Scully C, Bohorova N, Kim D, et al. (2011) Enhanced potency of a fucose-free monoclonal antibody being developed as an Ebola virus immunoprotectant. Proc Natl Acad Sci USA 108: 20690-20694.

6. Nimmerjahn F, Ravetch JV (2005) Divergent immunoglobulin G subclass activity through selective Fc receptor binding. Science 310: 1510-1512. 